PAPER

\title{
Second harmonic distortion suppression of thermoacoustic transducer using square root circuit
}

\author{
Takehiro Sugimoto* and Yoshiki Nakajima \\ Science \& Technology Research Laboratories, NHK, \\ 1-10-11 Kinuta, Setagaya-ku, Tokyo, 157-8510 Japan
}

(Received 13 August 2015, Accepted for publication 11 December 2015)

\begin{abstract}
Suppression of the second harmonic distortion intrinsic to a thermoacoustic transducer was studied using a square root circuit. The thermoacoustic transducer used in this study is composed of aluminum film, polyimide, and graphite film in order for it to be thin and bendable. A theoretical model was developed to calculate the radiated sound pressure on the basis of a fundamental equation for the heat transfer between the transducer and surrounding air. A square root circuit was designed to enable real-time signal processing and the effectiveness of the circuit regarding the second harmonic distortion suppression was experimentally confirmed.
\end{abstract}

Keywords: Thermoacoustic effect, Transducer, Harmonic distortion, Modeling

PACS number: 43.35.Ud, 43.38.Ar [doi:10.1250/ast.37.99]

\section{INTRODUCTION}

Sound is generated by mechanical vibration in common audio devices. It originates from the expansion and compression of the air induced by the vibration of a string or plate [1], and all commercially available loudspeakers are based on this principle. An example of sound generation without mechanical vibration is the thermoacoustic effect. Sound generated by this effect originates from the interaction between heat energy and acoustic energy. A temperature variation of substance in touch with the air generates a wave of condensation and rarefaction in the air [2], and this sound wave is independent of mechanical vibration.

One of the earliest studies on sound generation by the thermoacoustic effect was presented by Arnold and Crandall in 1917 [3]. They fabricated a thermophone composed of a raw platinum strip as a heat electrode and modeled its performance. Many recent studies on this effect have been carried out in the semiconductor field. Shinoda et al. proposed a thermoacoustic transducer adopting porous silicon as a heat insulator on a silicon substrate, and succeeded in ultrasonic emission [4]. Hu et al. modeled the thermoacoustic transducer proposed by Shinoda et al. [5], and Koshida et al. studied the digital operation of a thermoacoustic transducer composed of porous silicon [6]. Odagawa et al. developed printable

*e-mail: sugimoto.t-fg@nhk.or.jp semiconductor nanoparticles and confirmed thermally induced ultrasound emission from the device [7].

Several studies on improving the efficiency of thermoacoustic transducers have been reported this century, which were based on modification of the structure and adoption of advanced materials [8]. Boullosa and Santillan estimated the sound pressure radiated from thermoacoustic transducers made of a metal and various substrates by considering an equivalent velocity of pistonic motion [9]. Niskanen et al. proposed a suspended metal wire array to increase the contact surface of the heat electrode in a thermoacoustic transducer [10]. Vesterinen et al. modeled the suspended metal wire array and verified the model by comparing the results with experimental results [11]. Xiao et al. proposed a film loudspeaker made from carbon nanotubes and theoretically estimated its acoustical performance [12]. Suzuki et al. fabricates a heat electrode with a web structure from carbon nanotubes to increase the area in contact with the air [13].

The advantage of using a thermoacoustic transducer as a loudspeaker is vibration-free sound generation. Devices in which a vibration-free loudspeaker is required include flexible displays [14], which are lightweight, thin, and bendable, because the vibration of a conventional loudspeaker may cause flickering and blurring of images on the display and damage the internal circuits. Thus, we previously developed a flexible thermoacoustic transducer for integration with flexible displays [15] and reported its acoustical performance [16]. However, the radiated sound 
(a)

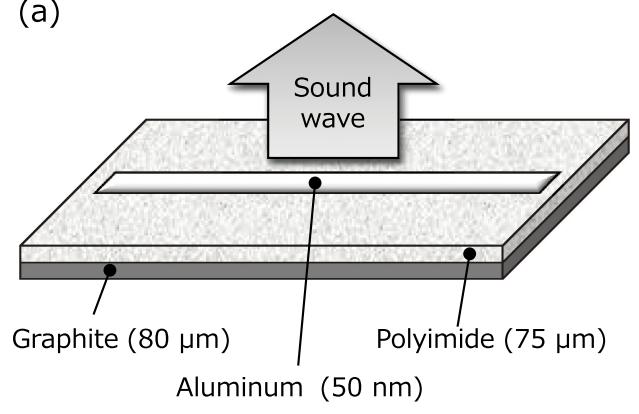

(b)

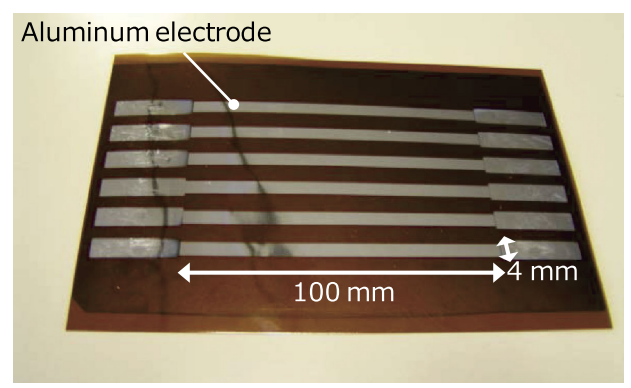

Fig. 1 (a) Schematic view of the thermoacoustic transducer. (b) Image of fabricated thermoacoustic transducer. Six transducers were fabricated on the same substrate, but only one transducer was used in this study.

pressure from the thermoacoustic transducer has double the frequency of the input signal. Such a property brings about the second harmonic distortion under conventional operation, which is superimposition of the ac audio signal on the dc bias voltage [16]. This problem remains to be solved.

In this paper, we propose a method of suppressing the second harmonic distortion intrinsic to the thermoacoustic transducer using a square root circuit. This circuit is designed to enable real-time signal processing. We also developed a theoretical model to calculate radiated sound pressure on the basis of a fundamental equation for the heat transfer between the transducer and surrounding air and the heat conduction equation. We experimentally confirmed the effectiveness of the square root circuit.

The rest of this paper is organized as follows. Section 2 outlines the thermoacoustic transducer used in this study. The harmonic distortion suppression method using the square root circuit is proposed in Sect. 3. The theoretical model of the thermoacoustic transducer is given in Sect. 4. Section 5 presents the experimental results and Sect. 6 provides a discussion of the results. Finally, a conclusion is given in Sect. 7.

\section{FABRICATED THERMOACOUSTIC TRANSDUCER}

Figure 1(a) shows a schematic view of our previously proposed thermoacoustic transducer [15]. It is composed of three thin films: an aluminum film that functions as an electrode for heat radiation to the air, a polyimide film as a heat insulator, and a graphite film as a heat sink, with thicknesses of about $50 \mathrm{~nm}, 75 \mu \mathrm{m}$, and $80 \mu \mathrm{m}$, respectively. This thermoacoustic transducer is sufficiently bendable, so it can be rolled up. Note that the fabricated thermoacoustic transducer used in this study was kept flat.

Figure 1(b) shows an image of the fabricated thermoacoustic transducer. Each aluminum electrode in the transducer is a $100 \mathrm{~mm} \times 4 \mathrm{~mm}$ strip. We fabricated six transducers on the same substrate then selected a single transducer for use in this study. We applied a voltage between both shorter edges of the electrode. The resistance between the edges of the heat electrode is $22 \Omega$. The reason we selected the strip shape is to uniformly distribute the current on the electrode because various paths with different lengths can be set between the terminals for a two-dimensional shape, e.g., circle or square. In such a case, the current concentrates on the path with the lowest resistance, which results in the entire area of the heat electrode not being efficiently used for heat generation.

\section{SECOND HARMONIC DISTORTION SUPPRESSION USING SQUARE ROOT CIRCUIT}

\subsection{Operating Principle of Thermoacoustic Trans- ducer}

The operating principle of the thermoacoustic transducer is based on the thermoacoustic effect of converting heat energy to acoustic energy. The energy conversion process starts from the generation of Joule heat on the heat electrode by inputting electrical power. Next, the generated heat is transferred to the air in contact with the electrode surface, heating the air. Then, the variation in heat causes a variation of the air density, resulting in a wave of condensation and rarefaction. Therefore, a sound wave is generated.

The left column of Fig. 2 illustrates a signal diagram of the input signal, Joule heat, and sound pressure in normal input. If we input only ac signal voltage to the thermoacoustic transducer, the generated Joule heat is modulated in accordance with the variation of input power, not input signal. The input power is proportional to the square of the input signal; thus, the frequency of the Joule heat is duplicated from that of the input signal $[3,15]$. Thus, the radiated sound pressure has double the frequency of the input signal, as shown in the left column of Fig. 2.

\subsection{Proposed Use of Square Root Circuit}

To avoid the duplication of the frequency mentioned 


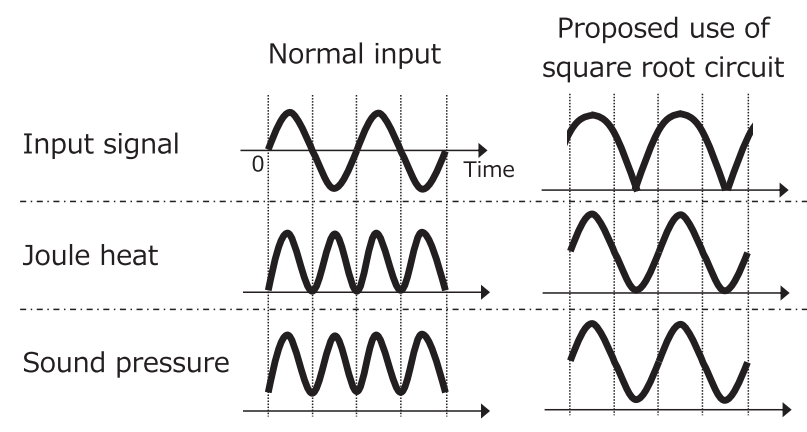

Fig. 2 Signal diagram of input signal, Joule heat, and sound pressure. Left column illustrates normal input, i.e., only ac signal is input. Right column illustrates using square root circuit.

in the former section, we superimposed an ac audio signal voltage on the dc bias voltage as the input signal in a previous study [16]. A positive input signal was maintained so that the frequency of the fundamental response of the sound pressure would not be duplicated. However, the second harmonic distortion increases proportionally to the square of the ac audio signal voltage.

We describe the above-mentioned phenomena assuming that the input signal $V_{\text {in }}$ is

$$
V_{\mathrm{in}}=V_{\mathrm{dc}}+V_{\mathrm{ac}} \sin \omega t
$$

where $V_{\mathrm{dc}}$ is the dc bias voltage, $V_{\mathrm{ac}}$ is the amplitude of the ac audio signal, $\omega$ is the angular frequency, then the sound pressure $p$ is in the following relationship:

$$
\begin{aligned}
p & \propto\left(V_{\mathrm{dc}}+V_{\mathrm{ac}} \sin \omega t\right)^{2} \\
& =V_{\mathrm{dc}}^{2}+\frac{V_{\mathrm{ac}}^{2}}{2}+2 V_{\mathrm{dc}} V_{\mathrm{ac}} \sin \omega t-\frac{V_{\mathrm{ac}}^{2}}{2} \cos 2 \omega t .
\end{aligned}
$$

The term $\cos 2 \omega t$ corresponds to the second harmonic distortion component, so that generation of the second harmonic distortion is inevitable. Moreover, the coefficient of $\cos 2 \omega t$ includes $V_{\mathrm{ac}}^{2}$, which may have a significant effect on audio reproduction because the distortion grows at double the rate of the reproduction level of the fundamental response.
Hence, we propose a method of suppressing the second harmonic distortion using a square root circuit. The concept is to prevent the ac audio signal from being squared and the frequency of the ac audio signal from being doubled. Specifically, it adopts the square root of the right side of Eq. (1) for the input signal as

$$
V_{\mathrm{in}}=\sqrt{V_{\mathrm{dc}}+V_{\mathrm{ac}} \sin \omega t}
$$

under the condition that $V_{\mathrm{dc}}+V_{\mathrm{ac}} \sin \omega t \geq 0$. In principle, the proposed method entirely suppresses the second harmonic distortion because the term including $\cos 2 \omega t$ is not produced in this case.

\subsection{Implementation of Square Root Circuit}

Figure 3(a) shows a block diagram of the square root circuit. We designed this circuit using the AD538AD realtime computational unit manufactured by Analog Devices, Inc. to enable real-time signal processing. The reason we developed the square root circuit by using a hardware processor is to easily measure and demonstrate the thermoacoustic transducer in real time.

Figure 3(b) is an image of the fabricated square root circuit. The square root circuit was implemented by NF Corporation. The frequency range of the ac audio signal is from dc to $50 \mathrm{kHz}$, the maximum amplitude of the ac audio signal is $17 \mathrm{~V}$, and the maximum dc bias voltage is $17 \mathrm{~V}$. These ranges were derived from the fact that the maximum applied voltage for the fabricated thermoacoustic transducer was $20 \mathrm{~V}$. Therefore, around $10 \mathrm{~V}$ is at least required for both the amplitude of the ac audio signal and the dc bias voltage so as not to double the frequency; thus, we set $17 \mathrm{~V}$ as the maximum value for both the ac audio signal and the dc bias voltage to leave a margin.

\section{THEORETICAL MODELING}

To analyze the radiated sound from the thermoacoustic transducer when using the square root circuit, we theoretically modeled it on the basis of a fundamental equation of the heat transfer between the transducer and surrounding air referred to in previous studies [3,12]. (a)

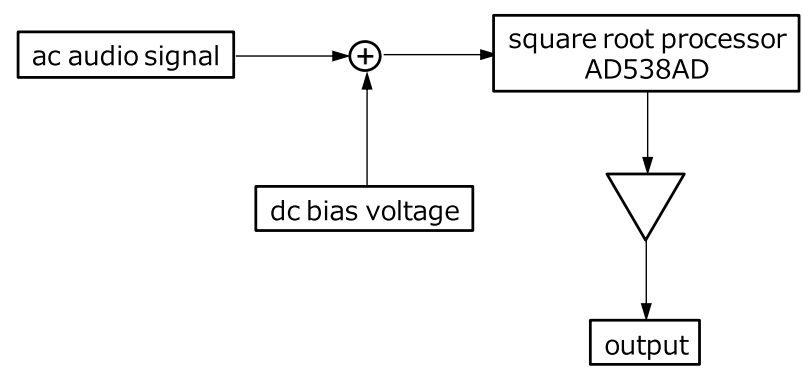

(b)

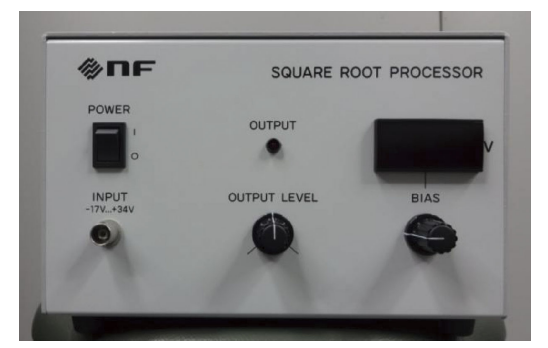

Fig. 3 (a) Block diagram of the square root circuit. (b) Fabricated square root circuit. 
The relationship between the applied voltage and temperature change of the heat electrode is described as follows:

$$
\begin{aligned}
\frac{\left(\sqrt{V_{\mathrm{dc}}+V_{\mathrm{ac}} \sin \omega t}\right)^{2}}{R} & =\frac{V_{\mathrm{dc}}+V_{\mathrm{ac}} \sin \omega t}{R} \\
& =\left(S+S_{\mathrm{g}}\right) h T+S C_{\mathrm{S}} \frac{d T}{d t},
\end{aligned}
$$

where $R$ is the resistance of the heat electrode, $S$ is the area of one side of the heat electrode, $S_{\mathrm{g}}$ is the area of the heat sink, $h$ is the rate of the heat loss per unit area, $C_{\mathrm{S}}$ is the heat capacity per unit area of the heat electrode, and $T$ is the temperature of the heat electrode above room temperature. The rate of the heat loss per unit area of the heat electrode and that of the heat sink were regarded as similar in this study because their surface and form are flat and common [12].

The heat conduction equation is a parabolic partial differential equation as follows:

$$
\frac{\partial T}{\partial t}=\alpha \frac{\partial^{2} T}{\partial x^{2}},
$$

where $\alpha$ is the thermal diffusivity of the air and $x$ is the distance from the surface of the thermoacoustic transducer in the vertical direction.

One solution to both Eqs. (4) and (5) is considered to be in the form of

$$
T(x, t)=B+A e^{-k_{T} x} \sin \left(\omega t-k_{T} x+\varphi\right) .
$$

Then, substituting Eq. (6) into Eq. (4) under the conditions that $\omega t=\pi / 2$ and $x=0, A$ is derived as

$$
A=\frac{V_{\mathrm{ac}}}{R\left\{h\left(S+S_{\mathrm{g}}\right) \cos \varphi-\omega S C_{\mathrm{S}} \sin \varphi\right\}} .
$$

In a similar manner, substituting Eq. (6) into Eq. (4) and comparing the dc and ac components, $B$ and $\varphi$ are derived as

$$
\begin{aligned}
B & =\frac{V_{\mathrm{dc}}}{h R\left(S+S_{\mathrm{g}}\right)}, \\
\varphi & =-\tan ^{-1} \frac{\omega S C_{\mathrm{S}}}{h\left(S+S_{\mathrm{g}}\right)} .
\end{aligned}
$$

Finally, substituting Eq. (6) into Eq. (5), $k_{T}$ is derived as

$$
k_{T}=\sqrt{\frac{\omega}{2 \alpha}} .
$$

The mean temperature increase $\tilde{T}$ caused by a temperature wave over a distance of one wavelength $\lambda_{T}$ is derived as follows:

$$
\tilde{T}=\frac{1}{\lambda_{T}} \int_{0}^{\lambda_{T}}(T-B) d x
$$

$$
\begin{aligned}
& =\frac{|A|}{\lambda_{T}} \int_{0}^{\lambda_{T}} e^{-k_{T} x} \sin \left(\omega t-k_{T} x+\varphi\right) d x \\
& =\frac{|A|\left(1-e^{-2 \pi}\right)}{2 \sqrt{2} \pi} \sin \left(\omega t+\varphi-\frac{\pi}{4}\right),
\end{aligned}
$$

where $\lambda_{T}=\frac{2 \pi}{k_{T}}=2 \pi \sqrt{\frac{2 \alpha}{\omega}}$.

In this system, a parameter $u$ equivalent to the displacement of the diaphragm of a loudspeaker is given in terms of $\lambda_{T}$ as follows considering $1 \gg e^{-2 \pi}$ [12]:

$$
\begin{aligned}
u & =\lambda_{T} \frac{\tilde{T}}{B+T_{0}} \\
& =\frac{|A|}{B+T_{0}} \sqrt{\frac{\alpha}{\omega}} \sin \left(\omega t+\varphi-\frac{\pi}{4}\right),
\end{aligned}
$$

where $T_{0}$ is room temperature.

In a similar manner to a vibrating plate, the velocity potential of the sound wave for a spherical distribution can be expressed as

$$
\Psi=-\left.\frac{S}{4 \pi r} \sin (\omega t-k r) \frac{\partial u}{\partial t}\right|_{\max },
$$

where

$$
\left.\frac{\partial u}{\partial t}\right|_{\max }=\frac{|A|}{B+T_{0}} \sqrt{\alpha \omega},
$$

$k$ is the wave number and $r$ is the distance from the thermoacoustic transducer. Thus, the sound pressure $p$ is derived as

$$
\begin{aligned}
p & =-\rho_{0} \frac{\partial \Psi}{\partial t} \\
& =\frac{\rho_{0} S|A| \sqrt{\alpha} \omega^{\frac{3}{2}}}{4 \pi r\left(B+T_{0}\right)} \cos (\omega t-k r),
\end{aligned}
$$

where $\rho_{0}$ is the density of the air. Therefore, rms value of the sound pressure $p_{\text {rms }}$ is given as a function of the frequency $f$ as follows:

$$
\begin{aligned}
p_{\mathrm{rms}}= & \frac{\sqrt{\pi} \rho_{0} S \sqrt{\alpha} V_{\mathrm{ac}}}{2 r\left\{V_{\mathrm{dc}}+h R\left(S+S_{\mathrm{g}}\right) T_{0}\right\}} \\
& \times \frac{f^{\frac{3}{2}}}{\sqrt{1+\left\{\frac{2 \pi S C_{\mathrm{S}}}{h\left(S+S_{\mathrm{g}}\right)}\right\}^{2} f^{2}}} .
\end{aligned}
$$

From Eq. (15), $p_{\text {rms }}$ proves to be proportional to $f^{\frac{1}{2}}$ in the frequency range much higher than $f_{0}$, which is given as

$$
f_{0}=\frac{h\left(S+S_{\mathrm{g}}\right)}{2 \pi S C_{\mathrm{S}}} .
$$

Figure 4 shows the fundamental response of the thermoacoustic transducer calculated using Eq. (15) plotted as the sound pressure level (SPL) in rms at the measurement point. The thermal properties and parameters used in the calculation were as follows: $V_{\mathrm{dc}}=10[\mathrm{~V}]$, 


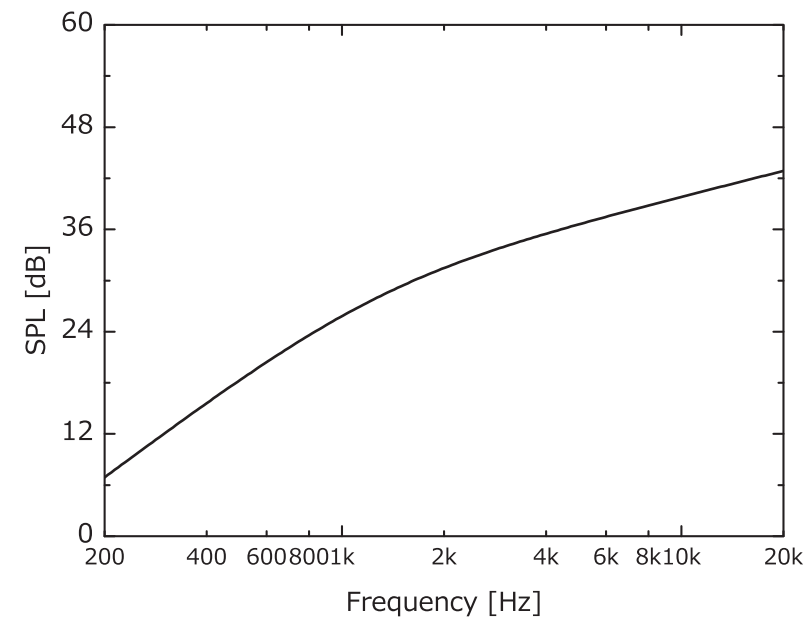

Fig. 4 Fundamental response of the thermoacoustic transducer calculated using Eq. (15).

$V_{\mathrm{ac}}=9.9[\mathrm{~V}], \quad R=22[\Omega], \quad r=0.5[\mathrm{~m}], \quad T_{0}=293[\mathrm{~K}]$, $\rho_{0}=1.2\left[\mathrm{~kg} / \mathrm{m}^{3}\right], \quad \alpha=2.2 \times 10^{-5}\left[\mathrm{~m}^{2} / \mathrm{s}\right], \quad h=25$ $\left[\mathrm{W} /\left(\mathrm{m}^{2} \cdot \mathrm{K}\right)\right][12], S=4.0 \times 10^{-4}\left[\mathrm{~m}^{2}\right], S_{\mathrm{g}}=1.5 \times 10^{-2}$ $\left[\mathrm{m}^{2}\right]$, and $C_{\mathrm{S}}=0.12\left[\mathrm{~J} /\left(\mathrm{m}^{2} \cdot \mathrm{K}\right)\right]$. These parameters $V_{\mathrm{dc}}$, $V_{\mathrm{ac}}, R, r, T_{0}, S$ and $S_{\mathrm{g}}$ were measured in this study, and common values were used for $\rho_{0}, \alpha$ and $C_{\mathrm{S}}$.

It is anticipated from Fig. 4 that the fundamental response of the fabricated thermoacoustic transducer has the point of inflection at around $1.2 \mathrm{kHz}$ calculated using Eq. (16).

\section{EXPERIMENTAL RESULT}

\subsection{Experimental Setup}

All measurements were conducted in an anechoic chamber with dimensions of $7.8 \mathrm{~m}(\mathrm{~W}) \times 7.6 \mathrm{~m}(\mathrm{D}) \times 6.8$ $\mathrm{m}(\mathrm{H})$ and a cut-off frequency of $40 \mathrm{~Hz}$. The noise rating (NR) of the anechoic chamber is 0 . Measurements were performed at a distance of $0.5 \mathrm{~m}$ from the thermoacoustic transducer, using a $1 / 2^{\prime \prime}$ free-field microphone (type-4191; Brüel \& Kjær) and a microphone amplifier (type-2669; Brüel \& Kjær). The thermoacoustic transducer was driven using a high-speed amplifier (HSA4101; NF Corporation). Neither baffles nor enclosures were used in these measurements.

All measurements were carried out using stepped sinusoidal waves with an interval of $1 / 80$ octave. A longer duration between 20 cycles and $10 \mathrm{~ms}$ was adopted as the signal duration for each frequency. Thus, the obtained results were averaged at least 20 times. In this experiment, SPL is in rms.

\subsection{Fundamental Response}

Figure 5 shows the fundamental response of the thermoacoustic transducer. The experimental results are illustrated as a solid line and the theoretical results

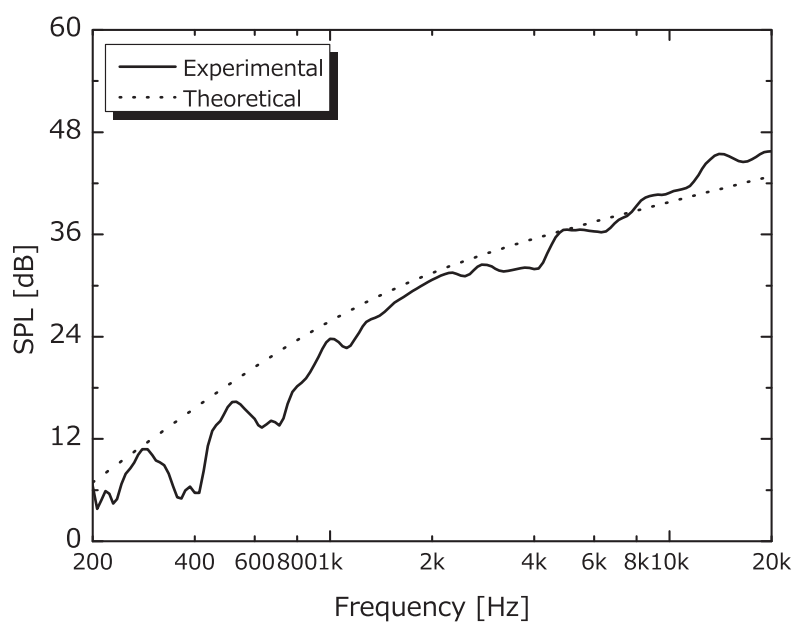

Fig. 5 Fundamental response of the thermoacoustic transducer. Experimental results are illustrated as solid line and theoretical results calculated using Eq. (15) are illustrated as dotted line.

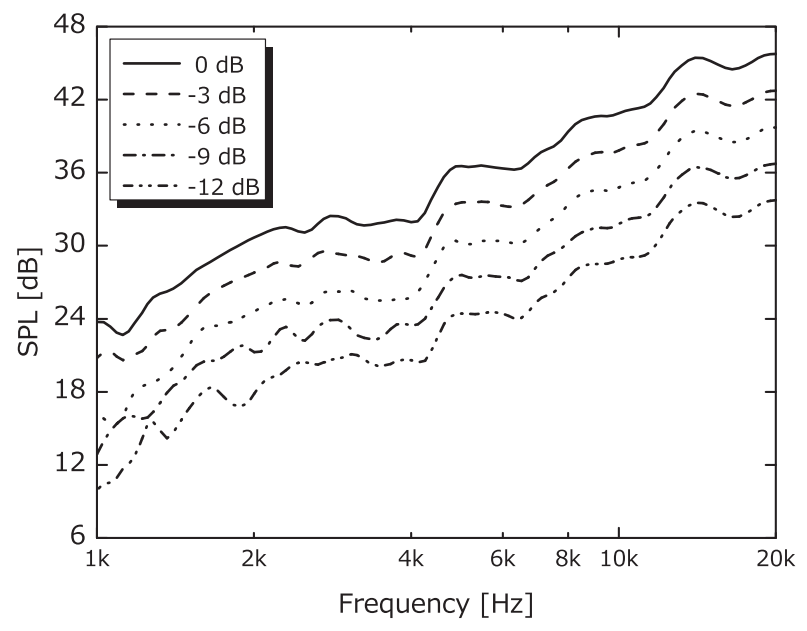

Fig. 6 Transition of fundamental response in 3-dB intervals of ac audio signal level. $0 \mathrm{~dB}$ corresponds to experimental result in Fig. 5.

calculated using Eq. (15) are illustrated as dotted line. The fabricated thermoacoustic transducer was driven under the condition in Fig. 4, namely $V_{\mathrm{dc}}=10[\mathrm{~V}]$ and $V_{\mathrm{ac}}=9.9$ [V]. Note that $V_{\text {ac }}$ of $9.9 \mathrm{~V}$ corresponds to $7 \mathrm{~V}$ in rms.

The theoretical results are generally in good agreement with the experimental results throughout the entire frequency range. In addition, the point of inflection $f_{0}$ is observed between 1 and $2 \mathrm{kHz}$, which agrees with the theoretical result of $1.2 \mathrm{kHz}$.

\subsection{Linearity}

We measured the linearity of the thermoacoustic transducer when using the square root circuit. Figure 6 shows a transition of the fundamental response when the ac audio signal level changed by $3 \mathrm{~dB}$. The frequency range 


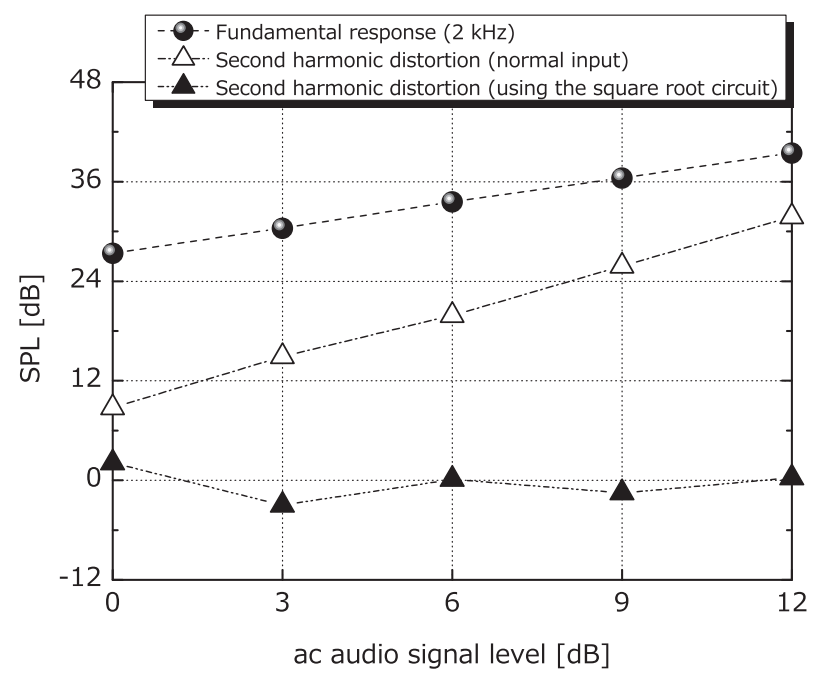

Fig. 7 SPL transition plotted as a function of the ac audio signal level in rms. The fundamental response at $2 \mathrm{kHz}$ (spheres) and the second harmonic distortion at $4 \mathrm{kHz}$ in normal input (white triangles) and using the square root circuit (black triangles) are plotted.

higher than $1 \mathrm{kHz}$ was measured because the signal-tonoise ratio $(\mathrm{S} / \mathrm{N})$ of the measurement deteriorated in the frequency range lower than $1 \mathrm{kHz}$. We selected the frequency range in which the $\mathrm{S} / \mathrm{N}$ ratio is sufficiently maintained throughout the entire level range to clarify the discussion on linearity. The result of $0 \mathrm{~dB}$ corresponds to the experimental result in Fig. 5. The level of $0 \mathrm{~dB}$ corresponds to the maximum input level for the fabricated thermoacoustic transducer. Thus, we can estimate around $20 \mathrm{~V}$ as the maximum applied voltage.

We found that the fundamental response generally increases proportionally to the amplitude of the ac audio signal. Therefore, we can safely say that the thermoacoustic transducer generates sound following the change of the ac audio signal, and the linearity of the fundamental response scarcely depends on the frequency.

\subsection{Second Harmonic Distortion Suppression}

We experimentally confirmed the effectiveness of the square root circuit by comparing both cases of normal input and using the square root circuit.

Figure 7 shows the SPL transition for the ac audio signal level in rms. The fundamental response at $2 \mathrm{kHz}$ (spheres) and the second harmonic distortion at $4 \mathrm{kHz}$ in normal input (white triangles) and using the square root circuit (black triangles) are plotted. The fundamental response levels were adjusted to reproduce sound at the same level under both conditions. In normal input, the dc bias voltage was fixed at $10 \mathrm{~V}$ and the ac audio signal voltage was $7 \mathrm{~V}$ in rms at the point on the horizontal axis with an ac audio signal level of $12 \mathrm{~dB}$. In using the square root circuit, the input signal is a positively biased non-

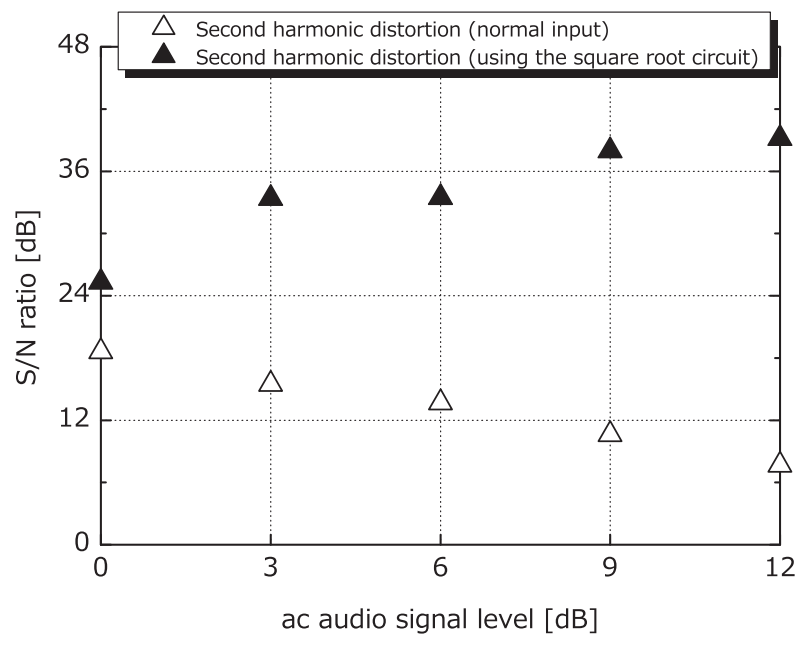

Fig. 8 The transition of $\mathrm{S} / \mathrm{N}$ ratio of the fundamental response at $2 \mathrm{kHz}$ to the second harmonic distortion at $4 \mathrm{kHz}$ plotted as a function of the ac audio signal level. The $\mathrm{S} / \mathrm{N}$ ratio in normal input (white triangles) and using the square root circuit (black triangles) are plotted.

sinusoidal ac signal with $14 \mathrm{~V}$ in rms at the point on the horizontal axis with an ac audio signal level of $12 \mathrm{~dB}$.

It can be seen from Fig. 7 that the fundamental response is proportional to the ac audio signal and the second harmonic distortion is proportional to the square of the ac audio signal in normal input. On the other hand, the second harmonic distortion level when using the square root circuit is approximately $0 \mathrm{~dB}$ regardless of the ac audio signal level. The value $0 \mathrm{~dB}$ was equivalent to the noise floor of the measurement system in this study, meaning that second harmonic distortion was not detected.

Figure 8 shows the transition of the $\mathrm{S} / \mathrm{N}$ ratio of the fundamental response at $2 \mathrm{kHz}$ to the second harmonic distortion at $4 \mathrm{kHz}$ and plotted as a function of the ac audio signal level. The $\mathrm{S} / \mathrm{N}$ ratio in normal input (white triangles) and using the square root circuit (black triangles) are plotted.

From Fig. 8, the $\mathrm{S} / \mathrm{N}$ ratio deteriorates in normal input as the ac audio signal level increases. In contrast, the $\mathrm{S} / \mathrm{N}$ ratio improves by using the square root circuit as the ac audio signal level increases.

Hence, we concluded that the proposed method successfully suppresses the second harmonic distortion as intended.

\section{DISCUSSION}

The theoretical results of the fundamental response were found to be in good agreement with that of the experimental results throughout the entire frequency range, as shown in Fig. 5. Thus, we concluded that the theoretical model is valid for calculating radiated sound pressure from the thermoacoustic transducer with the square root circuit, 
which in turn is useful to design thermoacoustic transducers.

The experimental results of the fundamental response deviated in the frequency range lower than $1 \mathrm{kHz}$. The sound radiation from the heat electrode of the strip shape remains for further discussion.

\section{CONCLUSION}

We proposed a method of suppressing the second harmonic distortion of the thermoacoustic transducer using the square root circuit. We also theoretically modeled the thermoacoustic transducer when using the square root circuit, and the derived model was proved to be valid. We confirmed from the experimental results that the second harmonic distortion was entirely suppressed by using the square root circuit. The results obtained in this study will lead to a design method for thermoacoustic transducers.

\section{REFERENCES}

[1] P. Morse and K. Ingard, Theoretical Acoustics (Princeton University Press, Princeton, 1986), pp. 95-226.

[2] F. Hunt, Electroacoustics (The Acoustical Society of America, USA, 1982), pp. 40-41.

[3] H. Arnold and I. Crandall, "The thermophone as precision source of sound," Phys. Rev., 10, 22-38 (1917).

[4] H. Shinoda, T. Nakajima, K. Ueno and K. Koshida, "Thermally induced ultrasonic emission from porous silicon," Nature, 400, 853-855 (1999).

[5] H. Hu, T. Zhu and J. Xu, "Model for thermoacoustic emission from solids," Appl. Phys. Lett., 96, 214101 (2010).

[6] N. Koshida, D. Hippo, M. Mori, H. Yanazawa, H. Shinoda and T. Shimada, "Characteristics of thermally induced acoustic emission from nanoporous silicon device under full digital operation," Appl. Phys. Lett., 102, 123504 (2013).

[7] A. Odagawa, A. Matsushita and M. Hashimoto, "Thermally induced ultrasound emission from printable semiconductor nanoparticles," J. Appl. Phys., 108, 076104 (2010).

[8] R. Venkatasubramanian, "Nanothermal trumpets," Nature, 463, 619 (2010).

[9] R. Boullosa and A. Santillan, "Ultrasound radiation from simple thermoacoustic transducers," Acta Acust. united Ac., 90, 277-284 (2004).

[10] A. Niskanen, J. Hassel, M. Tikander, P. Maijala, L. Grönberg and P. Helistö, "Suspended metal wire array as a thermoacoustic sound source," Appl. Phys. Lett., 95, 163102 (2009).

[11] V. Vesterinen, A. Niskanen, J. Hassel and P. Helistö, "Fundamental efficiency of nanothermophones: modeling and experiments," Nano Lett., 10, 5020-5024 (2010).

[12] L. Xiao, Z. Chen, C. Feng, L. Liu, Z.-Q. Bai, Y. Wang, L. Qian, Y. Zhang, Q. Li, K. Jiang and S. Fan, "Flexible, stretchable, transparent carbon nanotube thin film loudspeakers," Nano Lett., 8, 4539-4545 (2008).

[13] K. Suzuki, S. Sakakibara, M. Okada, Y. Neo, H. Miura, Y. Inoue and T. Murata, "Study of carbon-nanotube web thermoacoustic loud speakers," Jpn. J. Appl. Phys., 50, 01BJ10 (2011).

[14] Y. Nakajima, M. Nakata, T. Takei, H. Fukagawa, G. Motomura, H. Tsuji, T. Shimizu, Y. Fujisaki, T. Kurita and T. Yamamoto, "Development of 8-in. oxide-TFT-driven flexible AMOLED display using high-performance red phosphorescent OLED," J. SID, 22, 137-143 (2014).

[15] Y. Nakajima and T. Sugimoto, "Flexible sound generator based on thermoacoustic effect," IEEE Sensors 2012, pp. 1252-1255 (2012).

[16] T. Sugimoto and Y. Nakajima, "Acoustic characteristics of a flexible sound generator based on thermoacoustic effect," Proc. Meetings on Acoustics, 19, 030004 (2013).

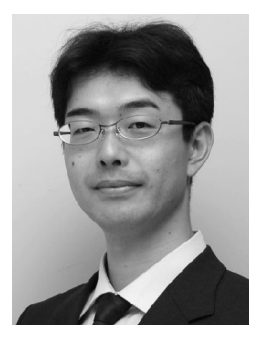

Takehiro Sugimoto received his B.E. and M.E. degrees in electronic engineering from the University of Tokyo, Tokyo, Japan, in 1999 and 2001, respectively. He also received a Ph.D. in information processing from Tokyo Institute of Technology, Tokyo, Japan, in 2013. He joined NHK in 2001 and has been working at Science \& Technology Research Laboratories since 2004. His research interests are acoustic transducers, audio coding, and three-dimensional sound system. He is currently engaged in MPEG and ARIB standardization.

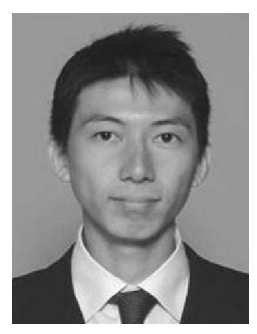

Yoshiki Nakajima received the B. Eng., M. Eng., and Dr. Eng. degrees in electrical and electronic engineering from Tokyo University of Agriculture and Technology, Tokyo, Japan, in 2000, 2002 and 2004, respectively. He joined NHK in 2004 and has been working at Science \& Technology Research Laboratories since 2005. He is currently working on development of the fabrication process and the driving technology of flexible displays and thermoacoustic devices. 